\title{
Control of AWD System for Vehicle Performance and Safety
}

\author{
Hojin Jung , Seibum Choi \\ KAIST, Department of Mechanical Engineering, 34141 Daejeon, Korea
}

\begin{abstract}
AWD (All-Wheel Drive) system transfers drive force to all wheels so that it can help vehicle escape low mu surface or climb hill more conveniently. Recently, AWD system for on road vehicle has become popular to improve vehicle driving performance. However, there has not been enough research of applying AWD system for vehicle stability especially for lateral movement. Compared with ESC (Electronic Stability Control), AWD system does not cause any inconveniences to the driver because it controls vehicle only by distributing front and rear drive torque, without using brake. By allowing slipping/locking of wet clutch inside the transfer case, AWD system can distribute different amount of torque between front and rear axle. This paper introduces modelling of AWD system and suggests the control of AWD system based on peak slip ratio and slip angle at which tyre saturates. Carsim based vehicle simulation results of AWD controller is presented.
\end{abstract}

\section{Introduction}

Compared with original $2 \mathrm{WD}$ vehicle which drives front wheels or rear wheels only, AWD system has a roll to distribute engine drive to torque to both front axle and rear axle. Usually AWD system was equipped with offroad vehicle that runs on rough road. However, with the growing of engine power, AWD system has become being equipped in high performance sedan.

Hardware configuration which is dealt with in this paper differs from center differential 4WD and selective 4 WD because it distributes some portion of drive torque of main drive shaft which is directly linked to transmission output shaft to sub drive shaft by controlling wet clutch inside the transfer case. Actuator type for control varies from ball ramp control type by electromagnetic force to hydraulic piston control type by motor pump.

By utilizing advantages of active control system, AWD system can be used for both of longitudinal and lateral driving safety. In this regard it is important to find out the present vehicle state as well as road state. For example, vehicle climbs high slope hill, escapes from low mu surface and has lateral movement like double lane change or circular motion with high velocity. And research about model predictive control should be done including these various driving situations. Then it is expected that vehicle performances like driver's riding quality and steady state cornering are improved.

This paper suggests the control method of AWD system of wet clutch with hydraulic piston actuator type. Vehicle simulation environment was set based on simulink built transfer case system coupled with carsim software. Especially, possibility of lateral stability improvement by AWD system without intervention of ESC is discussed in detail as well as longitudinal performance.

\section{System modelling}

\subsection{Driveline dynamic model}

States of AWD vehicle change depending on the amount of torque which is delivered front or rear shaft. AWD system which is dealt in this paper can control the amount of torque which is transfered to front drive shaft in transient state, by using multi plate wet clutch. Thus simulation description should be available of variable distriburtion of front shaft torque depending on clutch engagement force especially between clutch slipping state and clutch lock up state. Driveline model from transmission output shaft to front and rear differential gear can be represened as follows, mathematical model is also as follows :

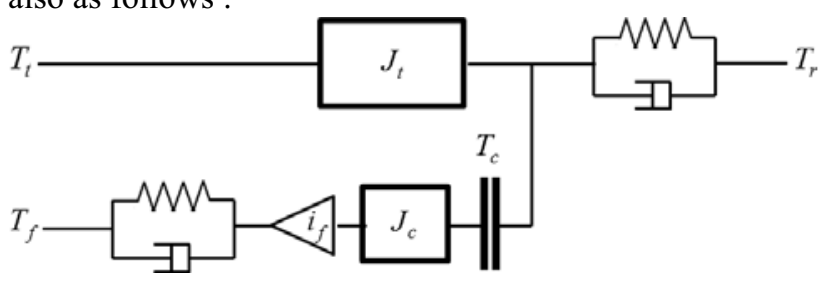

Figure 1. Schematic diagram of transfer case system.

$$
\begin{gathered}
T_{t}-T_{c}-T_{r}=J_{t} \dot{\omega}_{t} \\
T_{c}=f\left(F_{c}, \omega\right) \\
T_{c}-T_{f} / i_{f}=J_{c} \dot{\omega}_{c}
\end{gathered}
$$




$$
\begin{gathered}
T_{f}=k_{f}\left(\theta_{c}-\theta_{f}\right)+b_{f}\left(\dot{\theta}_{c}-\dot{\theta}_{f}\right) \\
T_{r}=k_{r}\left(\theta_{t}-\theta_{r}\right)+b_{r}\left(\dot{\theta}_{t}-\dot{\theta}_{r}\right)
\end{gathered}
$$

Where $T_{t}$ is a transmission output torque, $T_{c}$ is a clutch engagement torque, $T_{f}$ and $T_{r}$ are front and rear shaft torque each. $J_{t}$ is a main drive shaft inertia, $J_{c}$ is front drive shaft inertia, $F_{\mathrm{c}}$ is clutch engagement force given by hydraulic actuator. $\omega$ is a clutch slip angular velocity. Spring and damper system was added at each end of drive shaft to represent transfer torque given by distortion of shaft. Transmitted clutch torque is expressed as a function $f\left(F_{\mathrm{c}}, \omega_{\text {slip }}\right)$ and detailed expression is introduced in section 2.2.1.

\subsection{Wet clutch model}

Clutch is generally divided into dry clutch and wet clutch, depending on the kind of material and whether the oil is included or not. Dry clutch can be burned if slip duration prolonged. Wet clutch compensate the disadvantage of dry clutch, by absorbing the heat which is generated in friction plate. However, wet clutch is short of maximum allowable torque which friction plate endures. By overlapping multiple plates, maximum allowable torque can be enhanced.

\subsubsection{Generalized Maxwell Slip (GMS) friction model}

It is important to have a feasibility of friction model for the system which includes engagement force between two rolling plates to guarantee the validity of simulation. GMS model [1,2], which is well known for friction modelling, was opted for modelling transfer case system.

When clutch is fully lock up, differential equation of clutch transfer torque is as follows:

$$
\frac{d M_{i}}{d t}=k_{i}\left(p, T_{t}\right) \cdot \omega
$$

Where $k_{i}\left(p, T_{t}\right)$ is the function of clutch engagement force and transmission output torque. $k_{i}$ can be mapped by comparing carsim internal 4WD model front shaft torque and developed transfer case system front shaft torque.

When clutch is slipping, differential equation of clutch transfer torque is as follows:

$$
\frac{d M_{i}}{d t}=\operatorname{sgn}(\omega) C\left(\alpha_{i}-\frac{M_{i}}{s(\omega, p)}\right)
$$

Where $s(\omega, p)$ is a stribeck equation about torque, which is expressed as follows:

$$
s(\omega, p)=\operatorname{sgn}(\omega)\left(M_{s}+\left[M_{c}-M_{s}\right] e^{-\left|\frac{V_{s}(p)}{\omega}\right|}\right)
$$

Wet clutch should consider viscous effect by the oil film between disc plates. Especially for wet clutch, drag torque takes quite some part of transmitted torque so that this should be modelled carefully to improve the accuracy of transient response [4]. Drag torque of wet clutch is originated from the shear stress between plates and can be modelled by fluid dynamic analysis [4-6]. Then, viscous friction torque equation is obtained as follows:

$$
M_{v i s}=2 \pi \int_{r_{1}}^{r_{o}} \frac{\mu \omega r^{3}}{h}\left(1+0.0012 \operatorname{Re}_{h}^{0.94}\right) d r
$$

Where $r_{\mathrm{i}}$ is an clutch inner diameter, $r_{\mathrm{o}}$ is an effective diameter due to the centrifugal force, which is usually less than clutch outer radius. $\mathrm{Re}_{\mathrm{h}}$ is a Reynolds number Automatic Transmission Fluid (ATF), which is written as follows:

$$
\operatorname{Re}_{h}=\frac{\rho \omega r h}{\mu}
$$

Where $\mu$ is a viscous coefficient of ATF, $\rho$ is a density of ATF and $h$ is a clearance length of clutch plate.

Finally, clutch transfer torque when slipping is as follows:

$$
T_{c}=M_{i}+M_{v i s}
$$

To improve the accuracy of wet clutch engagement simulation, clutch experimental friction coefficient data which will be manufactured for vehicle implementation was used.

\subsection{Lock up state representation by applying DOF (Degree Of Freedom) reduction model}

GMS model which is introduced in section 2.2 matches well in normal driving simulation. However, complex or severe driving situation like rapid acceleration in low surface can cause model instability like chattering response of clutch transfer torque. Equation should be replaced when lock up state so that it was described by designing DOF reduction model. When lock up state, angular accelerations at both transmission output shaft and clutch driven shaft are same. Then, dynamic model of driveline is as follows:

$$
\begin{gathered}
\dot{\omega}_{t}=\dot{\omega}_{c} \\
T_{t}-T_{f}-T_{r}=\left(J_{t}+J_{c}\right) \dot{\omega}_{t} \\
T_{f}=k_{f}\left(\theta_{c}-\theta_{f}\right)+b_{f}\left(\dot{\theta}_{c}-\dot{\theta}_{f}\right) \\
T_{r}=k_{r}\left(\theta_{t}-\theta_{r}\right)+b_{r}\left(\dot{\theta}_{t}-\dot{\theta}_{r}\right)
\end{gathered}
$$

\subsection{State transition between clutch slipping and lock up}

It is important to decide the current state whether clutch is slipping or locked up to improve the accuracy of AWD system simulation [3]. GMS model distinguish current state only by the limit of allowable static torque. However, for the case of simulation which include full vehicle model. Above condition was not enough to determine state transition accurately when clutch is engaged while input torque is rapidly increasing. Thus two conditions of static torque and clutch slip were used simultaneously to enhance the accuracy of state transition.

$$
\begin{gathered}
T_{c} \geq M_{s} \\
\omega_{\text {slip }} \leq 0.05 \mathrm{rad} / \mathrm{s}
\end{gathered}
$$




\section{Controller design}

\subsection{Vehicle state estimation}

For the AWD system controller based on tyre model, current vehicle states should be estimated. Generally, the lowest wheel angular velocity value among 4 angular velocity sensors can be assumed to vehicle longitudinal velocity in $2 \mathrm{WD}$. However, when clutch is engaged, vehicle longitudinal velocity of AWD cannot be assumed only with wheel sensors because all wheels become driven wheels. Thus vehicle longitudinal velocity should be estimated. In addition, vehicle lateral velocity should be estimated to know wheel side slip angle. By utilizing vehicle longitudinal dynamics, lateral dynamics, yaw dynamics and wheel dynamics, these vehicle states can be estimated by extended Kalman filter $[7,8]$ using planar bicycle model shown in figure 2 .

The equation of motion for the bicycle model shown in figure 2 can be categorized three dynamics.

Longitudinal dynamics is:

$$
\begin{aligned}
& \dot{v}_{x}=\frac{1}{m}\left\{F_{x f} \cos \left(\delta_{f}\right)-F_{y f} \sin \left(\delta_{f}\right)+F_{x r} \cdots\right. \\
& \left.-\frac{1}{2} \rho_{\text {air }} C_{d} A v_{x}^{2}-m g \sin (\theta)+v_{y} r\right\}
\end{aligned}
$$

Where $v_{x}$ is the longitudinal velocity, $m$ is the vehicle mass, $F_{x f}$ is the front longitudinal axle force, $F_{y f}$ is the front lateral axle force, $\delta_{f}$ is the tire steer angle, $\rho_{\text {air }}$ is the air density, $\mathrm{C}_{d}$ is the drag coefficient, $v_{y}$ is the lateral velocity, $r$ is the yaw rate and $\theta$ is hill angle.

Lateral dynamics is:

$$
\dot{v}_{y}=\frac{1}{m}\left\{F_{x f} \sin \left(\delta_{f}\right)+F_{y f} \cos \left(\delta_{f}\right)+F_{y r}\right\}+v_{x} r
$$

Where $F_{y r}$ is the rear lateral axle force.

Yaw dynamics is:

$$
\dot{r}=\frac{1}{I_{z}}\left\{l_{f}\left(F_{x f} \sin \left(\delta_{f}\right)+F_{y f} \cos \left(\delta_{f}\right)\right)-l_{r} F_{y r}\right\}
$$

Where $I_{z}$ is the vehicle yaw moment of inertia, $l_{f}$ is the distance from the center of mass to the front axle and $l_{r}$ is the distance from the center of mass to the rear axle.

Another dynamics which force terms are related is wheel dynamics which is expressed as follows:

$$
\dot{\omega}=\frac{1}{I_{w}}\left\{T_{d}-T_{b}-R_{e} F_{x, i}-R_{e} F_{r}\right\}
$$

Where $\omega$ is the wheel angular velocity, $I_{w}$ is the wheel inertia, $T_{d}$ is the wheel drive torque, $T_{b}$ is the wheel brake torque. In this paper, $T_{d}$ is assumed to be known and does not consider braking situation. $R_{e}$ is the wheel effective radius, $F_{r}$ is the tire rolling resistance. $F_{x, i}$ is the each tire longitudinal force whose subscript $i$ includes $f l$ (front left), $f r$ (front right), $r l$ (rear left), $r r$ (rear right) which is written throughout the paper.

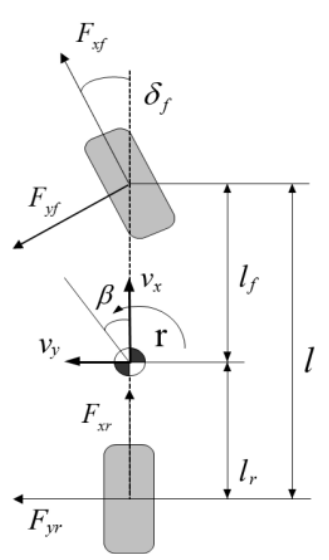

Figure 2. Bicycle model.

Using dynamics which are mentioned above, Kalman filter can be obtained. States of Kalman filter is defined as follows:

$$
\mathbf{x}(t)=\left[v_{x}, v_{y}, r, \boldsymbol{\omega}, \mathbf{F}\right]^{T}
$$

Where

$$
\begin{gathered}
\boldsymbol{\omega}=\left[\omega_{f l}, \omega_{f r}, \omega_{r l}, \omega_{r r}\right] \\
\mathbf{F}=\left[F_{x f l}, F_{x f r}, F_{x r l}, F_{x r r}, F_{y f}, F_{y r}\right]
\end{gathered}
$$

Tire longitudinal forces which are expressed in this paper are related as:

$$
F_{x f}=F_{x f l}+F_{x f r}, F_{x r}=F_{x r l}+F_{x r r}
$$

The measurements signals from vehicle are as follows:

$$
\mathbf{y}(t)=\left[a_{x}, a_{y}, r, \omega_{f l}, \omega_{f r}, \omega_{r l}, \omega_{r r}\right]
$$

Where $a_{x}$ and $a_{y}$ are related as:

$$
\begin{aligned}
& a_{x}=\frac{1}{m}\left\{F_{x f} \cos \left(\delta_{f}\right)+F_{y f} \sin \left(\delta_{f}\right)+F_{x r} \cdots\right. \\
& \left.-\frac{1}{2} \rho_{\text {air }} C_{d} A v_{x}^{2}-m g \sin (\theta)\right\} \\
& a_{y}=\frac{1}{m}\left\{F_{x f} \sin \left(\delta_{f}\right)+F_{y f} \cos \left(\delta_{f}\right)+F_{y r}\right\}
\end{aligned}
$$

The above states and measurements are expressed as nonlinear function $f(\mathbf{x}(t), \mathbf{u}(t))$ and $h(\mathbf{x}(t), \mathbf{u}(t))$ as follows:

$$
\begin{array}{r}
\dot{\mathbf{x}}(t)=f(\mathbf{x}(t), \mathbf{u}(t))+w(t) \\
y(t)=h(\mathbf{x}(t), \mathbf{u}(t))+v(t)
\end{array}
$$

Estimated states are usually denoted as $\hat{\imath}$. Then, the algorithms of discrete time extended kalman filter can be expressed as follows [10]:

$$
\begin{aligned}
& \hat{\mathbf{x}}_{k}(-)=\hat{\mathbf{x}}_{k-1}(+)+\int_{t_{k-1}}^{t_{k}} f(\mathbf{x}(\tau), \mathbf{u}(\tau)) d \tau \\
& \mathbf{P}_{k}(-)=\mathbf{P}_{k-1}(+) \cdots \\
&+\int_{t_{k-1}}^{t_{k}}\left\{\mathbf{F}(\tau) \mathbf{P}(\tau)+\mathbf{P}(\tau) \mathbf{F}^{T}(\tau)+\mathbf{L} \mathbf{L}^{T}\right\} d \tau \\
& \mathbf{K}_{k}(-)=\mathbf{P}_{k}(-) \mathbf{H}_{k}^{T}\left[\mathbf{H}_{k} \mathbf{P}_{k}(-) \mathbf{H}_{k}^{T}+\mathbf{R}_{k}\right]^{-1} \\
& \hat{\mathbf{x}}_{k}(+)=\hat{\mathbf{x}}_{k}(-)+\mathbf{K}_{k}\left[\mathbf{y}_{k}-h\left(\hat{\mathbf{x}}_{k}(-), \mathbf{u}(t)\right)\right] \\
& \mathbf{P}_{k}(+)=\left[\mathbf{I}-\mathbf{K}_{k} \mathbf{H}_{k}\right] \mathbf{P}_{k}(-)
\end{aligned}
$$


Where $F$ and $H$ are the linearization of equation (29) and (30) around $\hat{\mathbf{x}}_{k}(-)$ at each time step. $\mathbf{P}$ is the error covariance and $\mathbf{L}$ is the sensor noise disturbance matrix.

Wheel slip ratio and side slip angle are obtained from the estimated states. Longitudinal slip ratio at each wheel in acceleration is given by:

$$
\lambda_{i}=\frac{R_{e} \omega_{i}-\hat{v}_{x}}{R_{e} \omega_{i}}
$$

Also side slip angle of front axle and rear axle which will be used in the AWD logic development are:

$$
\begin{gathered}
\alpha_{f}=\beta+\frac{l_{f} r}{\hat{v}_{x}}-\delta_{f} \\
\alpha_{r}=\beta-\frac{l_{r} r}{\hat{v}_{x}}
\end{gathered}
$$

Where $\beta$ is side slip angle which is defined as $v_{y} / v_{x}$.

\subsection{AWD driving logic development}

Certainly, AWD driving condition can be decided based on saturation point of tyre longitudinal and cornering stiffness. AWD system has a tendency to stabilize the vehicle both of longitudinal and lateral movements. There many approaches to develop vehicle control logic based on tyre stiffness. However, there has not been saturation point detection logic considering vehicle weight shifting and slip simultaneously. To solve this problem tyre lateral force function of two variables about vertical force and slip angle is presented here.

$$
\begin{gathered}
F_{x}=C_{x, 1} \lambda_{i}+C_{x, 2} \lambda_{i}^{2} \\
F_{y}=C_{y, 1}\left(1-\frac{\Delta F_{z}}{F_{z, n}}\right) \alpha+C_{y, 2} \alpha^{2}
\end{gathered}
$$

Where $F_{z, n}$ is nominal vertical force of normal load. $\Delta F_{z}$ is the difference between shifted vertical force and nominal vertical force.

Saturation point of slip ratio and slip angle can be calculated by differentiating equation (39) and (40).

$$
\begin{gathered}
\lambda_{\text {sat }}=-\frac{C_{x, 1}}{2 C_{x, 2}} \\
\alpha_{\text {sat }}=-\frac{C_{y, 1}}{2 C_{y, 2}\left(1-\frac{\Delta F_{z}}{F_{z, n}}\right)}
\end{gathered}
$$

Above saturation point is used in this paper to determine the engagement of wet clutch.

\section{Simulation}

To validadte the AWD system driving simulation by impementing developed transfer case plant model, carsim software was used. As shown in figure 2, simulink based transfer case model was coupled in carsim. Except for transfer case part carsim internal model was used. Simulation coupled with commercial sofware not only can verify the versatility of developed model but also help to easiliy grasp of change of vehicle state depending on various driving simulation. Vehicle driveline is based on front ship rear wheel drive (FR) type.

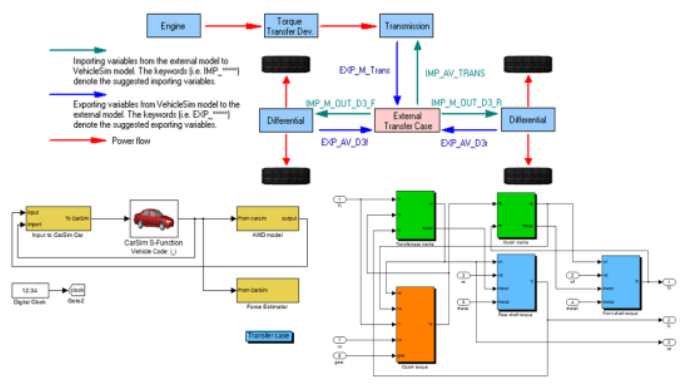

Figure 3. Transfer case simulink model coupled with carsim.

\subsection{Accelerating in split mu situation}

For the validation of longitudinal driving performance of AWD system, straight line with split mu simulation was conducted. Left wheel is driven on the low mu with friction coefficient of 0.2 and right wheel is driven on the high mu with friction coefficient of 0.85 . While driving split mu with $30 \mathrm{~km} / \mathrm{h}$, rapid full acceleration was conducted between 8-14 seconds to simulate the situation of high slip ratio. Based on the slip calculation logic and trigger condition which was mentioned in section 3.2 and 3.3, AWD system was activated. To exclude the influence of gear shifting, only $1^{\text {st }}$ and $2^{\text {nd }}$ gear shifting was allowed.
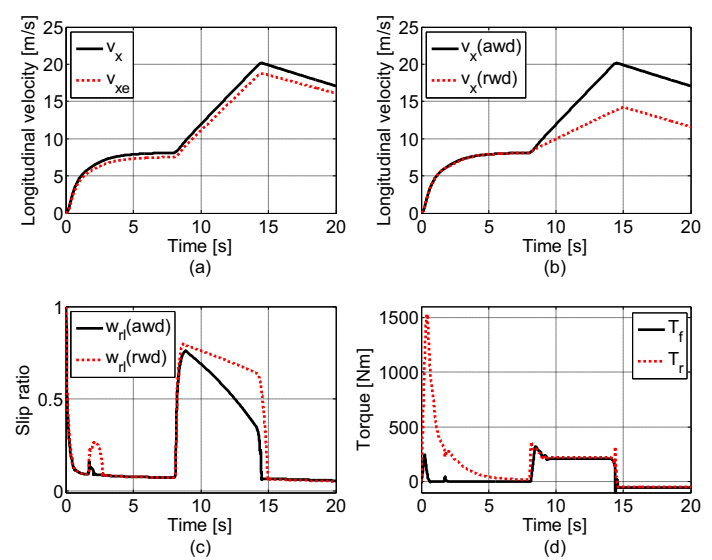

Figure 4. Simulation results of accelerating in split mu situation. (a) Comparison of longitudinal velocity between real value and estimated value (b) Comparison of longitudinal velocity between awd and rwd (c) Comparison of slip ratio between awd and rwd (d) front and rear shaft torque response in awd

Figure 4(a) shows the results of estimated awd longitudinal velocity. Although there appeared discrepancy between real value and estimated value, it can be used for slip ratio calculation. And figure 4(b) shows the results of longitudinal velocity between awd and rwd. Certainly, awd showed a better performance to escape the split mu road. Slip ratio reduction in awd compared to rwd can be verified in figure 4(c). Figure 4(d) shows the torque response of awd by the control logic. 


\subsection{Accelerating in steady state cornering situation}

For the validation of lateral stability of AWD system, 500 feet radius circle lane simulation was conducted. While driving high mu of friction coefficient with $90 \mathrm{~km} / \mathrm{h}$, rapid full acceleration was conducted between 15-25 seconds to simulate the situation of oversteering. Then, AWD system was activated based on same process which was mentioned in section 4.1. Maximum transmission gear was set to $5^{\text {th }}$.

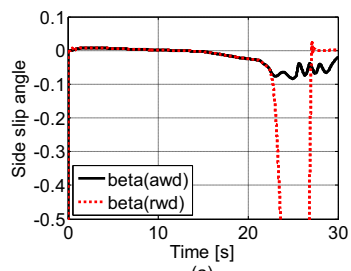

(a)

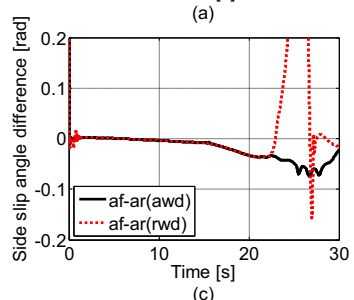

(c)
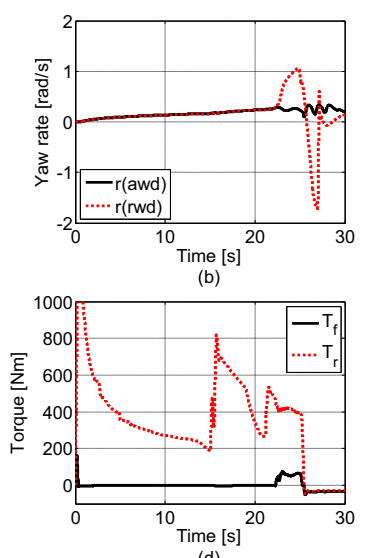

Figure 5. Simulation results of accelerating in steady state cornering. (a) Comparison of side slip angle between awd and rwd (b) Comparison of yaw rate between awd and rwd (c) Comparison of front and rear slip difference between awd and rwd (d) front and rear shaft torque response in awd

Figure 5(a) and 5(b) shows the results of side slip angle and yaw rate, which are principal indices of lateral stability, between awd and rwd. Certainly, rwd showed high side slip angle and yaw rate when tyre saturates. Figure 5(c) shows the results of front and rear axle side slip angle difference. Actually, awd showed slight side slip angle difference, which means vehicle moves similar to neutral steer. However, rwd showed an over-steering response when tyre force saturates. Figure 5(d) shows the torque response of awd by the control logic. It is noteworthy that small amount of torque distribution prevented over-steering response.

\section{Conclusion}

AWD control logic development for vehicle stability was conducted in this paper. For controller validation, transfer case modeling method was introduced and reliable simulation environment was built first, by implementing simulink transfer case as an external model in carsim software. Noticable reuslt of simulation is that upper level controller based on tyre force function of wheel slip ratio and slip angle helped tyre not to fall into saturation region, without intervention of ESC system. In addition, small amount of torque transfer in subdrive shaft stabilize the vehicle lateral movement efficiently. However, explanation of some principal vehicle states estimator is omitted by assuming they are measurable in this paper. Thus it should be furthur studied for practical application. Also, real time tyre stiffness and mu estimation based logic will improve the awd control logic for various driving scenario.

\section{Acknowledgement}

This work was supported by the National Research Foundation of Korea(NRF) grant funded by the Korea government(MSIP) (No. 2010-0028680).

This research was supported by the MSIP(Ministry of Science, ICT and Future Planning), Korea, under the CITRC(Convergence Information Technology Research Center) (IITP-2015-H8601-15-1005) supervised by the IITP(Institute for Information \& communications Technology Promotion)

\section{References}

1. Agusmian Partogi Ompusunggu, Paul Sas, Hendrik Van Brussel, "Modeling and simulation of the engagement dynamics of a wet clutch system subjected to degradation: An application to condition monitoring and prognostics", Mechatronics (2013)

2. Vincent Lampaert, Farid Al-Bender, Jan Swevers, "A Generalized Maxwell-Slip Friction Model appropriate for Control Purposes", IEEE Physics and Control Proceedings. International Conference (2003)

3. Jiwon J. Oh, Seibum B. Choi, Jinsung Kim, "Driveline modeling and estimation of individual clutch torque during gear shifts for dual clutch transmission", Mechatronics (2014)

4. Hiromu HASHIMOTO, Sanae WADA and Yoshiaki MURAYAMA, "An Influence of Centrifugal Force on the Performance of Thermo-hydrodynamic Turbulent Thrust Bearings", Bulletin of JSME, 27, No. 228 (1984)

5. Yiqing Yuan, Eysion A. Liu, James Hill, Qian Zou, “An Improved Hydrodynamic Model for Open Wet Transmission Clutches", ASME Journal of Fluids Engineering (2007)

6. Youhei Takagi, Yasunori Okano, Masatoshi Miyayaga and Nobuyuki Katayama, "Numerical and Physical Experiments on Drag Torque in a Wet Clutch", Japanese Society of Tribologists, pp. 242-248 (2012)

7. Laura R. Ray, "Nonlinear state and tire force estimation for advanced vehicle control", IEEE TRANSACTIONS ON CONTROL SYSTEMS TECHNOLOGY, 3, NO.1 (1995)

8. Mooryong Choi, Jiwon J. Oh and Seibum Choi, "Linearized Recursive Least Square Methods for Realtime Identification of Tire-Road Friction Coefficient", IEEE TRANSACTIONS ON VEHICULAR TECHNOLOGY, 8, (2013) 